\title{
PROKLA-Redaktion
}

\section{Editorial: Materialistischer Feminismus}

Seit einigen Jahren lässt sich beobachten, dass vielfach ein Kulturkampf um das Geschlecht stattfindet. Dieser betrifft unter anderem heterosexuelle Männer, gleichgeschlechtliche Paare und Transgender. Auch feministisch bewegte Frauen sind in besonderer Weise betroffen, denn sie haben seit den 1960er Jahren in der Zweiten Frauenbewegung gegen die sie betreffenden Praktiken der Herrschaft, also gegen die spezifischen Formen der vergeschlechtlichenden Unterwerfung und herrschaftlichen Erzeugung ihrer Subjektivität gekämpft, gegen die moralische, medizinische, staatliche Kontrolle ihres Körpers, den Sexismus oder die Ausbeutung in den Familien oder am Arbeitsplatz. Der Kulturkampf richtet sich in vielerlei Hinsicht gegen diese Emanzipation. Anders Breivik gab als einen der Gründe für sein Massaker unter norwegischen jungen Sozialist_innen an, dass seine Aktion auch eine gegen Feminismus und "Genderismus "1 gerichtete sein sollte. Frauen haben in Italien gegen ihre Entwürdigung demonstriert, dass sie von den Vertretern des Staates selbst zu Sexualobjekten erniedrigt werden, in Spanien kämpfen sie darum, dass eine vergleichsweise liberale Abtreibungsregelung von der konservativen

1 Dieser Begriff wurde von katholischen Fundamentalisten und rechtsradikalen Kreisen eingeführt, um die Bezugnahme auf Gender als Familien zerstörend und Männer diskriminierend zu diskreditieren.
Regierung nicht verschärft wird. In Polen lehnt der Klerus eine Entschädigung der Opfer der priesterlichen Missbrauchspraktiken mit der abstrusen Konstruktion ab, wonach eigentlich der Genderismus Ursache dafür gewesen sei, dass die Jugendlichen es den Priestern leicht gemacht hätten. In der Schweiz werden feministische und Gendertheorien von überregionalen Zeitungen und konservativen Politiker_innen als Irrlehren betrachtet. In diesem Zusammenhang stehen auch Auseinandersetzungen um die Frage der Sexualaufklärung von Jugendlichen in Deutschland und der Schweiz. Entgegen den Versuchen der Normalisierung, derzufolge die Frauen doch heute gleich gestellt seien, müssen Frauen erfahren, dass sie im Haushalt weiterhin mehr arbeiten, ihre Doppelbelastung höher ist und dass ihre Einkommen und Karrierechancen schlechter sind als die von Männern. Weiterhin werden viele Frauen, aber vor allem auch junge Mädchen, im privaten und öffentlichen Raum mit Belästigung, Abwertung bis hin zu sexualisierter Gewalt konfrontiert.

Materialistischer Feminismus nimmt umfassend gesellschaftstheoretisch und in emanzipatorischer Perspektive die Gesamtheit der Verhältnisse in den Blick, unter denen Geschlechterungleichheit (re)produziert wird, sich verschiedene Herrschaftsverhältnisse verschränken, aber auch Möglichkeiten des Widerstands hervorgerufen werden: „Feminismus lässt sich 
als Ensemble von Debatten, kritischen Erkenntnissen, sozialen Kämpfen und emanzipatorischen Bewegungen fassen, das die patriarchalen Geschlechterverhältnisse, die alle Menschen beschädigen, und die unterdrückerischen und ausbeuterischen gesellschaftlichen Mächte, die insbesondere Frauenleben formen, begreifen und verändern will." (Hennessy 2003: 155) Als ein solches Ensemble sind feministische Ansätze somit immer beides: Sie sind Teil einer Theoriebildung sowie einer im weitesten Sinn verstandenen politischen Praxis, die in gesellschaftliche Verhältnisse intervenieren wollen. Gleichzeitig werden unter dem Begriff Feminismus heterogene Konzepte gefasst, die sich geschichtlich wie inhaltlich differenzieren lassen. Bekannt geworden ist der Begriff durch die Schriften des Sozialphilosophen und Frühsozialisten Charles Fourier, der den Grad der Befreiung der Frau als Prüfstein einer jeden Gesellschaft und allgemeinsten Maßstab der menschlichen Entwicklung formuliert hat. Dabeiging es den Frühfeminist_innen wie Olympe de Gouges oder Mary Wollstonecraft zunächst vor allem um die Etablierung gleicher Rechte wie den Persönlichkeitsrechten in der Ehe, dem Scheidungs-, Wahl- oder dem Recht auf Eigentum und Bildung. Dass darüber hinaus auch schon in den 1860er Jahren die Frage nach der Umgestaltung von Hausarbeit in verschiedensten feministischen Programmen im Mittelpunkt stand, wird von Katharina Volk in ihrem Beitrag für das vorliegende Heft aufgezeigt. Die sogenannten materiellen Feministinnen (material feminists) - zu denen nicht nur Sozialistinnen, sondern auch liberale Feministinnen gehörten sahen die Hausarbeit als eine wesentliche Ursache der Geschlechterhierarchie an (vgl. Federici 2012: 36).

Diese Analyse fand innerhalb der Ersten Frauenbewegung ebenfalls Anklang.
Sozialist innen wie Alexandra Kollontai forderten in der Sowjetunion der 1920er Jahre eine umfassende „Aufwertung und Vergesellschaftung der Hausarbeit“ (ebd.). Doch Kollontais Vorstellungen machten nicht bei der Hausarbeit halt, es ging ihr um nicht viel weniger als die Befreiung der Frau aus ihrem "Liebesgefängnis“ (Haug 2006: 83). Die romantische Liebe, die Monogamie und das Ehegelübde wurden von Kollontai als Relikte der bürgerlichen Gesellschaft betrachtet, die in ihren vermeintlich letzten Atemzügen lag. In ihrem aus Gesprächen und Interviews zusammengestellten Werk SecondhandZeit deutet die Schriftstellerin Swetlana Alexijewitsch an, welchen großen Einfluss Kollontais Schriften auf interessierte Sozialist_innen gehabt haben muss: „Wir sprachen von der Revolution und von der Liebe [...] wir stritten auch viel über das damals populäre Buch von Alexandra Kollontai Die Liebe der Arbeitsbienen. Sie verteidigte die freie Liebe, das heißt, die Liebe ohne alles Überflüssige [...] 'Wie ein Glas Wasser trinken' [...] Ohne Seufzer und ohne Blumen, ohne Eifersucht und Tränen“ (Alexijewitsch 2013: 197). Dass das nicht immer im Sinne einer „feministischen Befreiung “ ablief, lässt sich u.a. aus Robert Cohens historischem Roman Exil derfrechen Frauen ablesen. Die Sozialistin Maria Osten, die im Schatten ihres Geliebten, des großen Starjournalisten der Pra$w d a$, Michail Kolzow, stand, lässt Cohen sagen: „Freie Liebe, eine offene Beziehung, das war es doch, wozu Alexandra Kollontai die Genossinnen ermutigte, die sich von den Beengungen der bürgerlichen Ehe befreiten. Über den Preis, den die Frauen dafür bezahlten, sagte Kollonotai wenig." (Cohen 2009: 63) Interessant ist dabei, dass die innerhalb feministischer Strömungen durchaus kontrovers diskutierte Theoretikerin Judith Butler eine ähnliche 
Beobachtung für die Zweite Frauenbewegung macht: „Ich erinnere mich an die Geschichten über radikale Sozialistinnen, die Anfang der 70er Jahre die Monogamie und die Familienstruktur ablehnten und am Ende jenes Jahrzehnts vor den psychoanalytischen Praxen Schlange standen, um sich in Schmerzen auf der Couch zu winden" (Butler 2001: 121). Egal wie dieser Umstand nun interpretiert wird - Butler nennt es den ,utopische[n] Versuch der Mißachtung von Verboten" (ebd.) - und wie jeweils der Umfang und die Aussichten der Emanzipation verstanden werden, so zeigt er, dass auch Körper, Gefühle, Sexualität, Begehren Gegenstände materialistisch-feministischer Theorie und Praxis sein müssen. Oft beschränkt sich materialistischer Feminismus hingegen auf ein klassisches Thema, das mit einem reduzierten Verständnis von Materialismus einhergeht: Arbeit, Reproduktion und ökonomische Ungleichheit. Jede neue Generation oder Bewegung von Feministinnen wird um ihre eigenen - bzw. ihre vielen - Antworten in all diesen Bereichen ringen müssen. Aktuell verwundert es nicht, dass - nach einem Jahrzehnt der Show Americas next topmodel - gerade das Buch Fleischmarkt der Bloggerin Laurie Penny großen Anklang findet. Darin verhandelt sie den Begriff des 'erotischen Kapitals' und untersucht, wie sich innerhalb des Neoliberalismus' ganze Industrien um den weiblichen Körper gruppieren. Penny gilt der Körper somit als fundamentaler Bestandteil der Arbeits- und Kapitalstrukturen, die die weltweite Produktion überhaupt erst ermöglichen (vgl. Penny 2012: 119). Gegen die permanente Zurschaustellung des weiblichen Körpers richten sich immer wieder feministische Initiativen wie jüngst die Gruppe Pinkstinks, die dem Staffelstart von Germanys next Topmodel 2014 den Slogan 'Einfach top - ohne Model' entgegensetzte. Doch es gibt auch Interventionen innerhalb der Kulturindustrie selbst, die feministische Beachtung finden. In der HBO-Serie Girls von Lena Dunham wird mit der Figur der Hannah Horvarth eine weibliche Protagonistin portraitiert, die sich als Gegenmodell zu Heidi Klums 'Mädchen', welche öffentlich vor Millionen von Zuschauer_innen mit dem Maßband ausgemessen werden, präsentiert. Dunham, die Horvath gleichzeitig auch darstellt, wurde scharf kritisiert, zu oft ihren nackten Körper - der nicht zu den Schönheitsidealen der gängigen Serienformate passt - zu zeigen. Horvath unterwirft sich dem Diktat der Shapewear nicht (vgl. Schütt 2013). Sicher, eine reine Kritik auf der Ebene der Repräsentation, die innerhalb der Kulturindustrie verhandelt wird, ist für eine materialistisch-feministische Theorie zu wenig. Das bedeutet im Umkehrschluss allerdings nicht, dass sie irrelevant wäre.

Doch zurück zu den Forderungen der Zweiten Frauenbewegung. Diese nahm viele Impulse der Ersten wieder auf, spitzte die Kritik an patriarchalen Gesellschaftsstrukturen weiter zu und entwickelte neue Fokussierungen und Politikformen: Die Akteurinnen diskutierten Arbeits- und Lebensbedingungen von Frauen, insbesondere die geschlechtsspezifische Arbeitsteilung von Reproduktions- und Erwerbsarbeit, die Darstellung des weiblichen Körpers in den Medien, den Umgang mit dem eigenen Körper, mit Reproduktion und Sexualität sowie die bis dahin weitgehend tabuisierte (auch sexuelle) Gewalt gegen Frauen und Mädchen, insbesondere im Nahbereich von Ehe und Familie.

Sozialistische Feministinnen interessierten sich dabei vor allem dafür, wie das Verhältnis von Kapitalismus und Geschlechterverhältnissen zu denken sei, da sie davon ausgingen, dass Frauenunterdrückung untrennbar mit der Geschichte des 
Kapitalismus zusammenhängt. Als in diesem Zusammenhang einflussreichste Debatte kam in den frühen 1970er Jahren die 'Hausarbeitsdebatte' unter marxistischen Vorzeichen auf und machte Reproduktionsarbeit zum Hauptthema. Darin wurde die geschlechtsspezifische Arbeitsteilung als materielle Basis der Unterordnung von Frauen gesehen und die unbezahlte, unsichtbare und wie eine Naturressource 'selbstverständlich' verfügbare Hausarbeit ins Zentrum der Theoretisierungsversuche von Frauenunterdrückung im Kapitalismus gerückt (Asenbaum/Kinzel 2010). Kritik gab es zunächst an der marxschen Werttheorie und dem in ihr enthaltenen Arbeitsbegriff, dass unentlohnte Frauenarbeit nämlich nicht nur gesamtgesellschaftlich weitgehend unsichtbar, sondern auch in der marxschen Theorie systematisch zum Verschwinden gebracht sei (Haug 2004: 51). Dabei standen sich Ansätze, die Hausarbeit als Teil der kapitalistischen Produktionsweise sahen, sowie Ansätze, welche die Hausarbeit als eine nicht-kapitalistische häusliche Produktionsweise auffassten, gegenüber. Der sog. „Bielefelder Ansatz“ hingegen berief sich nicht auf werttheoretische Überlegungen, sondern behauptete unter Bezugnahme auf Rosa Luxemburgs Akkumulationstheorie Frauenunterdrückung als fortwährende ursprüngliche Akkumulation des Kapitals, d.h. als notwendige innere Kolonie, ohne die kapitalistisches Wachstum nicht möglich sei. Claudia von Werlhof, Maria Mies und Veronika Bennholdt-Thomsen sahen die Subsistenz- und nicht die Warenproduktion als die eigentliche Grundlage der Wertschöpfung. Dass der Zusammenhang von Kapitalismus und Geschlecht in der Hausarbeitsdebatte über das Verhältnis von bezahlter Lohnarbeit und unbezahlter Reproduktionsarbeit hergestellt wurde, ist dabei kein Zufall, sondern im Kontext des
Familienernährermodells mit der dazugehörigen geschlechtsspezifischen Arbeitsteilung sowie der politischen Aktivitäten der neuen Frauenbewegung zu sehen, die darauf gerichtet waren, die Familie als Ort von Gewalt, Herrschaft und Ausbeutung zu thematisieren und die im Haushalt verrichtete unbezahlte Reproduktionsarbeit als Arbeit sichtbar zu machen. Den Zusammenhang von Kapitalismus und Gender jedoch ausschließlich oder vorwiegend über das Verhältnis bezahlter und unbezahlter Lohn- und Hausarbeit zu betrachten, reicht als Anspruch einer feministisch-materialistischen Analyse nicht aus. Wie bereits die aufkommenden Fragen der ersten und zweiten Welle der Frauenbewegungen zeigen, spielen Themen wie Körper, generative Praktiken, Gesundheit, Liebe, Sexualität und Begehren eine ebensolche Rolle für den Zusammenhang von Geschlecht und kapitalistischer Produktionsweise, weil sie ein nicht unwesentlicher Teil ihrer wechselseitigen Konstitution sind.

Zwar brach der Streit um das Verhältnis von Kapitalismus und Geschlecht oder um eine Verbindung von Marxismus und Feminismus Ende der 1980er Jahre ab, jedoch haben die neueren Analysen und Forschungen zur Care-Ökonomie ihre Wurzeln in der Debatte der Neuen Frauenbewegung zur Haus- und Beziehungsarbeit, in dem also, was als Verbindung von Marxismus und Feminismus und als feministische Ökonomiedebatte begann. Obschon sich diese seit den 1980er Jahren sehr verändert hat, ist das analytische Problem, mit dem sich Theoretiker_innen der Neuen Frauenbewegung intensiv befasst haben - nämlich die Frage, wie sich die Bedeutung der unbezahlten Arbeit (ökonomisch) fassen lässt und worin der spezifische Charakter dieser (personenbezogenen Dienstleistungs-) Arbeit besteht - bestehen geblieben (vgl. Madörin 2007). Während der Begriff der 
Reproduktion für die marxistisch geprägte Hausarbeitsdebatte zentral war, hat sich in der Zwischenzeit eine Verschiebung von 'Reproduktion' zu 'Care' ergeben, die auch vor dem Hintergrund der Veränderungen in der Ökonomiekritik und der feministischen Kritik zu sehen ist. So war in der Hausarbeitsdebatte die Frage zentral, welche Rolle die Hausarbeit für die Kapitalakkumulation spielt, d.h. vor allem ob sie, da sie Arbeitskraft und Reproduktionsmittel herstellt, auch Mehrwert produziert oder ob sie nur indirekt zur Mehrwertproduktion beiträgt, indem sie die Arbeitskraft (und dazu noch billig) reproduziert. Mit dem Begriff und den Diskussionen um 'Care' rückte hingegen der spezifische Charakter häuslicher Arbeit, also die Für(einander)sorge, und somit emotionale, psychologische und Beziehungsfragen stärker in den Fokus. Es geht also darum, daraufhinzuweisen, dass CareArbeit auf Grund der im Zentrum stehenden Subjekt-Subjekt-Beziehungen anders betrachtet werden muss als z.B. Arbeit in der Güterproduktion: Da das Produkt dieser Tätigkeiten die Arbeit selbst sei, die aus den Beziehungen zwischen den involvierten Subjekten besteht und somit von der Arbeiter_in, die sie verrichtet, nicht zu trennen ist, spielen neben fachlicher Kompetenz die Beziehungskompetenz, die gegenseitigen Gefühle, die Kommunikation und die Qualität der Beziehung eine wichtige Rolle, sie alle sind Teil der Leistung und der Qualität der Leistung. Produktivitätsfortschritte haben auf Grund der nur beschränkt und nur für einige Care-Tätigkeiten möglichen Leistungsmessungen und Standardisierungen enge Grenzen, wenn die Beziehungen und somit die Qualität der Leistung nicht darunter leiden soll.

Neben dem Fokus der Analyse seit dem Ende der Hausarbeitsdebatte in den 1980er
Jahren hat sich auch die gesellschaftliche Organisation von Care durch zahlreiche Veränderungen gewandelt wie der Zunahme von Frauenerwerbstätigkeit, gestiegene Bildungsansprüche, veränderte Ansprüche an die Qualität von Beziehungen, höhere Lebenserwartungen, steigende Komplexität in der Gesundheitsversorgung oder neue Pflegeanforderungen und -bedarfe. Die gegenwärtigen feministischen Debatten um Care konstatieren eine im Zuge dieses Wandels entstehende umfassende Krise, die Care in allen seinen Facetten wie der Fürsorge, Erziehung, Pflege und Unterstützung, bezahlt und unbezahlt, in Einrichtungen und in privaten Lebenszusammenhängen betrifft. Dabei werden verschiedene Momente als krisenhaft beschrieben: entstehende Reproduktionslücken in den Privathaushalten, Versuche der Bearbeitung durch Delegation von Hausarbeit an Dritte - zumeist migrantische und illegalisierte Frauen -, Abbau staatlicher Zuwendungen und ein dadurch verschärfter Mangel an Personal im Bereich Pflege, Gesundheit und Erziehung oder die Zerstörung von Fürsorglichkeit durch Rationalisierungsstrategien in kommodifizierter Care-Arbeit. Mit der Debatte um diese Krise, die sowohl unter dem Begriff der Care-Krise wie unter dem der Reproduktionskrise kursiert, kehrt die Frage nach einer Theoretisierung von Care-Tätigkeiten und ihren kapitalistischen Zusammenhängen vermehrt zurück in feministische Auseinandersetzungen. Wie Care-Tätigkeiten theoretisch gefasst werden können, ist eine Frage, mit der sich Beatrice Müller in diesem Heft beschäftigt. Im Zuge der gegenwärtigen Situation der Krise werden mit den Diskussionen zu den Veränderungen von Care jedoch auch Fragen rund um den Krisenbegriff aufgeworfen. Inwiefern sich die Zuspitzungen und Verschiebungen im Bereich privat geleisteter oder bezahlter Care-Arbeit als 'Krise' fassen lassen und 
worin das Krisenhafte besteht, diskutiert Julia Dück in ihrem Beitrag.

Obwohl das Spektrum der Fragen sich nach der Hausarbeitsdebatte erweitert hat und mit dem Aufkommen des Care-Begriffs auch emotionale, psychologische und Beziehungsfragen vermehrt in den Blick geraten sind, bleiben die Perspektiven oft dennoch verengt und ihrem 'Teilbereich' verhaftet: Diskussionen um die Veränderungen oder die Krise von Care fokussieren häufig auf Veränderungen in den Arbeitsverhältnissen und die geschlechtsspezifische Arbeitsteilung. Ein Verschmelzen vielfältiger feministischer Erkenntnisse zum Beispiel durch die Integration weiterer geschlechtlicher $\mathrm{Di}$ mensionen in die Analysen gelingt nur zum Teil. Dennoch gibt es diese Brückenschläge: So versucht bspw. Antke Engel eine Verbindung verschiedener Perspektiven, indem sie queerfeministische Ansätze ${ }^{2}$ mit einer Kritik der politischen Ökonomie zu einer Analyse von Sexualität im Neoliberalismus zusammen denkt (vgl. Engel 2009).

An dem Beispiel der Verschiebung von Care-Tätigkeiten zu migrantischen Arbeiterinnen zeigt sich auch, dass eine feministisch-materialistische Analyse immer die rassistischen Dynamiken innerhalb einer Gesellschaft mitdenken muss, wie dies Encarnación Gutiérrez-Rodríguez mit dem Bezug auf Affekttheorie hier vorstellt. Auf die Blindstelle 'Rassismus' innerhalb feministischer Theorien, haben des weiteren insbesondere Schwarze ${ }^{3}$ Frauen bzw.

2 Queer Theory befasst sich mit der Frage, wie Körper, Geschlecht und Sexualität gedacht und gelebt werden können, ohne dass sie immer wieder an eine rigide ZweiGeschlechter-Ordnung und die Norm der Heterosexualität rückgebunden werden (Engel 2009: 19).

3 Die Bezeichnung Schwarz mit großem Anfangsbuchstaben soll den Konstruktcharakter
Women of Color - vor allem der Dritten Welle der Frauenbewegung - hingewiesen. Dabei wurde auch betont, dass 'der' Schwarze Mann historisch nie die gleiche Stellung innerhalb des Kapitalismus und des modernen Staats einnehmen konnte wie 'der' weiße Mann (Hennessy 2000: 66). Um eine ähnliche Autorität innerhalb des Familiengefüges einnehmen zu können, habe er weder über die symbolischen noch materiellen Ressourcen verfügt. Diese Argumentation führte zu einer anderen Analyse bisher genannter feministischer Schlüsselbegriffe wie Reproduktion oder eben auch Familie. Die Feministin Hazel Carby argumentiert in diesem Zusammenhang, dass die Schwarze Familie durchaus eine wichtige Widerstandsfunktion gegenüber kolonialer Unterdrückung übernommen habe und somit die Kritik weißer Feminist_innen an der Kernfamilie nicht in jeder Hinsicht zu teilen sei (Carby 1997: 112). Im Kontext der Auseinandersetzungen um verschiedene Formen der Unterdrückung ist im US-amerikanischen Black Feminism der späten 1970er und frühen 1980er Jahre der Begriff Intersektionalität entstanden. Ausgangspunkt war hierbei die Kritik Schwarzer Feministinnen an einem großen Teil der damaligen feministischen Bewegung, dass der Blick vieler weißer Feministinnen ausschließlich auf die Interessen weißer, heterosexueller Frauen gerichtet sei und hiermit die Vorstellung eines homogenen Subjekts Frau begründet, welche Differenzen zwischen Frauen und Herrschaftsverhältnisse jenseits von Geschlechterverhältnissen außer Acht ließe. Die Auseinandersetzungen mit den

der Kategorie 'Rasse' deutlich zu machen. Um im Kontext von Rassismus die privilegierende Bedeutung der Bezeichnung zu betonen, wird weiß hier hingegen kursiv geschrieben (Eggers u.a. 2005: 13). 
Differenzen und der unterschiedlichen Betroffenheit von Frauen hat somit gezeigt, dass Feminismus über die Beschäftigung mit patriarchalen Herrschaftsstrukturen hinaus auch andere Formen der Unterdrükkung in den Blick nehmen muss. Die Zusammenhänge verschiedener Herrschaftsverhältnisse herauszuarbeiten, bleibt dabei jedoch eine weiterhin bestehende Aufgabe gegenwärtiger feministischer Beschäftigung. Mit der unterschiedlichen Verwundbarkeit von Subjekten durch globale Prozesse - vor allem im Bereich der globalen Umweltveränderungen - beschäftigt sich Sarah Hackfort in ihrem Beitrag.

Rassistische Strukturen müssen von einer feministisch-materialistischen Analyse aktuell vor allem auch vor dem Hintergrund eines globalisierten Neoliberalismus, der zu einer „Neuzusammensetzung der globalen Arbeiterklasse“ geführt hat, in welche Frauen oft zu katastrophalen Bedingungen ,geschleudert" wurden (Haug 2006: 103), begriffen werden. Während im globalen Norden die Lohnarbeitsverhältnisse von Frauen vor allem im Dienstleistungssektor angesiedelt sind, ist im globalen Süden der Anteil an Frauen in den Exportfabriken besonders hoch. Dies bedeutet meist „Hungerlöhne, sehr lange Arbeitszeiten, Rechtlosigkeit, Gewaltdrohungen, mangelnde Sicherheitsvorkehrungen, keinerlei Chancen aufOrganisierung" (Holland-Cunz 2003: 201). Silvia Federici schlägt in diesem Zusammenhang vor, den globalisierten Neoliberalismus als einen Prozess der 'Rekolonisierung' zu betrachten (Federici 2012: 60). In diesem Zusammenhang muss auch die Frage nach der Gewalt gegenüber Frauen nochmal neu gestellt werden. So argumentiert bspw. die Sozialökonomin und Geschlechtertheoretikerin Vibhurti Patel, dass es einen $\mathrm{Zu}$ sammenhang zwischen dem Anstieg von Vergewaltigungen und der Verschärfung neoliberaler Politik in Indien gibt (vgl. Patel 2014: 5).

Wie die Debatten um eine Umgestaltung von Hausarbeit sich - wenn auch in gewandelter Form - in den verschiedenen feministischen Wellen fortsetzten, haben die Auseinandersetzungen mit Geschlechterverhältnissen immer wieder auch zu einer Beschäftigung mit Weiblichkeits- und Männlichkeitskonstruktionen geführt. Während zunächst 'nur' die Natürlichkeit geschlechtlicher Zuweisungen zu Denk-, Fühl- und Handlungsmustern in Frage gestellt wurde, hat Judith Butler mit ihrem Buch Das Unbehagen der Geschlechter Anfang der 1990er Jahre das Verständnis von Geschlecht als materielles Verhältnis erweitert. Mit der Aufhebung der Trennung von sex (biologisches Geschlecht) und gender (soziales Geschlecht) und ihrer Neuformulierung von Geschlecht als einem performativen Akt ist Butler zu einer wichtigen Stichwortgeberin (queer) feministischer Debatten geworden. Dabei ließe sich zwischen der Theorie und der 'Figur' Butlers unterscheiden, um die sich ein wahres Kampffeld entwickelt hat. Oft einseitig rezipiert, geht es Butler dabei nicht um einen „linguistischen Monismus“ (Butler 1997: 27), sondern um Analyse von Macht Foucaultscher Art, die sich materialisiert und in den Körper einschreibt.

Butler wurde dennoch durchaus scharf kritisiert, so bspw. von Rosemary Hennessy, die argumentiert, dass mit Butler ein post-marxistischer Feminismus Einzug halte, der symptomatisch für eine ganze Generation linker Theoriebildung sei. Hennessy kritisiert dabei vor allem, dass die materialistische Analyse einer Analyse des Symbolischen gewichen sei. Sie stellt dem historischen Materialismus einen akademisch vorherrschenden kulturalistischen Materialismus gegenüber. Der kulturalistische Materialismus enthalte 
auch kapitalismuskritische Elemente, sei aber andererseits durch die kulturellen Strategien und Formierungen des Spätkapitalismus leicht in denselben zu integrieren (vgl. Hennessy 2000: 28). Selbst wenn man diese Beobachtung teilt, muss das nicht zwingend bedeuten, dass man die Theorien von Butler und vielen anderen Autor_innen verwirft. Es ist sicherlich richtig, dass Butler keine Analyse kapitalistischer Strukturen vorlegt, auch - und da hat Hennessy einen sehr starken Punkt - wird Sexualität nicht innerhalb eines Systems der Ausbeutung und der Arbeit gedacht, das heißt allerdings nicht, dass Butlers Überlegungen, die sich um Fragen psychischer Repräsentanz und Möglichkeiten von Identitätsbildung drehen, nicht auch wesentliche Punkte einer materialistisch-feministischen Theorie der Gesellschaft berühren.

Die 'Figur' Butler gilt auch der Strömung des New Materialist Feminism als ein zentraler Abgrenzungspunkt. In ihrer 1988 erschienenen Schrift Situated Knowledges. The Science Question in Feminism and the Privilege of Partial Perspective entwickelt die Biologin Donna Haraway das Konzept des 'Situierten Wissens', welches von jeglichen Universalitätsansprüchen des Wissens absieht. Zudem wird 'Materie' nicht als 'passiv' oder 'tot' begriffen, sondern selbst als Agent bzw. als Akteur, womit Haraway den New Materialism vorbereitet. Der New Materialism bezieht sich einerseits auf die Philosophie von Gilles Deleuze und Félix Guattari (Elisabeth Grosz, Rosi Braidotti) und andererseits auf naturwissenschaftliche Konzepte wie die Physikphilosophie von Niels Bohr. Den Theorien des New Materialism ist gemeinsam, dass sie sich als radikal antianthropozentrisch verstehen und eine $\mathrm{Ab}$ kehr von jeglichem humanistischen Denken einfordern. Vielmehr noch: Der Tod der Kategorie Mensch - den Foucault einst beschwor - ist im Geflecht der technischen Möglichkeiten bereits eingetreten. Die Philosophie, die hinter dem New Materialism steht, sagt den vertrauten Instrumenten der Gesellschaftstheorie - Dialektik, Negativität, Kritik - den Kampf an. Ob der New Materialism dann noch in der Lage ist Herrschaft zu denken, erörtert Pia Garske in ihrem Beitrag zu diesem Band.

Das hier aufgemachte Spektrum feministischer Beschäftigung hat uns auch bei der Planung dieses Heftes begleitet. Dabei ging es uns nicht darum, die teilweise praktizierten Abgrenzungen zwischen marxistischen, poststrukturalistischen oder queertheoretischen feministischen Ansätzen zu wiederholen, sondern Möglichkeiten für produktive Verbindungen zu suchen. Wir halten solche Konfrontationen in vieler Hinsicht für überholt und überflüssig. Fragen nach rechtlicher Gleichstellung, geschlechtlicher Arbeitsteilung, Familie, Körperlichkeit, Liebe, Begehren oder Sexualität als vielfältige Dimensionen von Geschlechterverhältnissen müssen somit immer auch Teil materialistisch-feministischer Theorie und Praxis und materialistischer Gesellschaftstheorie sein, die zur Umwälzung aller Verhältnisse beitragen will, in denen Menschen beherrscht, ausgebeutet, erniedrigt, gequält und verlassen sind. Um die jeweiligen Erkenntnisse in einer Analyse gesamtgesellschaftlicher Verhältnisse fruchtbar zu machen, emanzipatorische Veränderungen gesellschaftlicher Verhältnisse also unter Einbezug der vielfältigen Dimensionen von Geschlechterverhältnissen zu betrachten, sind die verschiedenen Aspekte und ihre jeweiligen theoretischen Verortungen nicht gegeneinander zu stellen, sondern ist die Vielfältigkeit feministischer Beschäftigung wahrzunehmen.

Für die Redaktion: Julia Dück und Mariana Schütt 


\section{Literatur}

Alexijewitsch, Swetlana (2013) Seconhand-Zeit. Leben auf den Trümmern des Sozialismus. München.

Asenbaum, Maria/Kinzel, Katharina (2010): Wert und Wettex. In: Perspektiven. Magazinfür linke Theorie und Praxis, Nr. 9/2010, unter: http://www.perspektiven-online. at/2010/01/20/wert-und-wettex/, abgerufen am 08.01.2014.

Butler, Judith (1997): Körper von Gewicht. Frankfurt/M.

- (2001): Antigones Verlangen: Verwandtschaft zwischen Leben und Tod. Frankfurt/M.

Carby, Hazel V. (1982) (1997): White Woman Listen! Black Feminism and the Boundaries of Sisterhood in: Hennessy, Rosemary und Chris Ingraham: Materialist Feminism. A Reader in Class, Difference, and Women's Lives. New York/Abington, S. 110-128.

Cohen, Robert (2009): Exil der frechen Frauen. Berlin

Eggers, Maureen Maisha/Kilomba, Grada/ Piesche, Peggy/Arndt, Susan (2009): Konzeptionelle Überlegungen. In: dies. (Hg.): Mythen, Masken und Subjekte. Kritische Weißseinsforschung in Deutschland, Münster: 11-13.

Hennessy, Rosemary (2000): Profit and Pleasure. Sexual identities in Late Capitalism. New York/Abington.

Holland-Cunz, Barbara (2003): Die alte neue Frauenfrage. Frankfurt/M.

Engel, Antke (2009): Bilder von Sexualität und Ökonomie. Queere kulturelle Politiken im Neoliberalismus. Bielefeld.

Federici, Silvia (2012): Aufstand aus der Küche. Reproduktionsarbeit in globalen
Kapitalismus und die unvollendete feministische Revolution, Münster

Haug, Frigga (2004): Sozialistischer Feminismus: Eine Verbindung im Streit. In: Becker, Ruth/Kortendiek, Beate (Hrsg.): Handbuch Frauen-und Geschlechterforschung. Theorie, Methoden, Empirie, Wiesbaden, S.49-55.

- (2006): Marxistische Theorien und feministische Debatten. In: Nichoj, Torsten und Marco Tullney (Hrsg.): Geschlechterverhältnisse in der Ökonomie. Marburg, S. 73-120.

Hennessy, Rosemary (2000): Profit and Pleasure. Sexual identities in Late Capitalism. New York/Abington.

- (2003): Feminismus. In: Haug, Frigga (Hrsg.): Historisch-kritisches Wörterbuch des Feminismus. Hamburg, S.155-170.

Madörin, Mascha (2007): Neoliberalismus und die Reorganisation der Care-Ökonomie. In: http://www.denknetz-online. ch/IMG/pdf/Madorin.pdf, abgerufen am 14.01.2014.

Patel, Vibhurti (2014). Das dominante Konzept von Sexualität in Frage stellen! In: izw3 Februar 2014, 5 .

Penny, Laurie (2012): Fleischmarkt. Weibliche Körper im Kapitalismus. Hamburg

Schmiedel, Stevie (2014): Neun Jahre Topmodel sind genug. Im Mai sagen wir „Byebye, GNTM!“. In: http://pinkstinks.de/neunjahre-topmodel/, abgerufen am 09.02.2014.

Schütt, Mariana (2013): It was so good, I almost came. „Girls“ und die neue Ernsthaftigkeit. In: Prager Frübling No. 16, 52.

Thiessen, Barbara (2004): Feminismus: Differenzen und Kontroversen. In: Becker, Ruth/ Kortendiek, Beate (Hrsg.): Handbuch Frauen-und Geschlechterforschung. Theorie, Methoden, Empirie, Wiesbaden, S.35-41. 